\title{
PROTON EMISSION FROM GAMOW RESONANCE
}

\author{
T. VERTSE AND A. T. KRUPPA \\ Institute of Nuclear Research of the Hungarian Academy of \\ Sciences, H-4001 Debrecen, P.O. Box 51, Debrecen, Hungary \\ B. BARMORE \\ Department of Physics \& Astronomy, University of Tennessee, \\ Knoxville, Tennessee 37996, USA \\ Joint Institute for Heavy Ion Research, Oak Ridge, Tennessee \\ 37831, USA \\ W. NAZAREWICZ \\ Institute of Theoretical Physics, Warsaw University, Hoża 69, \\ PL-00-681 Warsaw, Poland \\ Department of Physics 83 Astronomy, University of Tennessee, \\ Knoxville, Tennessee 37996, USA \\ Physics Division, Oak Ridge National Laboratory, Oak Ridge, \\ Tennessee 37831, USA
}

AND

L. GR. IXARU AND M. RIZEA

Institute of Physics and Nuclear Engineering "Horia Hulubei", P.O. Box MG-6, Bucharest, Romania

\section{Introduction}

Our aim is to descibe proton emission from a deformed nucleus in a microscopic way. In our model, we assume that the shapes of the parent and the daughter nuclei are the same, and that both nuclei perform collective rotation. The parent state is constructed from collective states of a rotating core and single-proton wave functions. It is assumed that the proton moves in a quasi-bound state of an axially symmetric deformed potential. In the asymptotic region, the behavior of the proton wave function is governed by the $Q_{p}$ value of proton decay.

The proton resonance is described by a complex-energy Gamow state, i.e., the pole of the $S$-matrix on the complex energy (or $k$ ) plane, $\mathcal{E}_{n}=$ 
$E_{R}-i \frac{\Gamma}{2}$. The wave function of a Gamow state is regular at the origin and, at large distances, is propotional to a purely outgoing Coulomb wave

$$
u_{i}\left(r, k_{n}\right) \sim O_{l}\left(\eta_{n}, k_{n} r\right),
$$

where $k_{n}=\kappa-i \gamma\left(k_{n}^{2}=\frac{2 \mu}{\hbar^{2}} \mathcal{E}_{n}\right)$ is the complex wave number and $\eta_{n}$ is the Sommerfeld parameter. For a Gamow state, the decay width is simply $\Gamma=-2 \times \operatorname{Im}\left(\mathcal{E}_{n}\right)$; hence the half-life time of the single particle resonance is $\hbar \ln 2 / \Gamma=T_{1 / 2}^{s . p}$. For proton emitters from a spherically symmetric nucleus, the single particle picture works very well if one takes into account the many-body aspects by multiplying the single particle width by the spectroscopic factor $\mathcal{S}$ and comparing the measured $\Gamma_{\text {exp }}$ to $\mathcal{S} \Gamma[1]$.

\section{Deformed Gamow states in an adiabatic approximation}

Let us consider a single particle Hamiltonian $h=t+v(\boldsymbol{r})$ with an axially symmetric potential in the intrinsic frame of reference. In our calculations, for the average potential we use a deformed optical Woods-Saxon potential (with the spherical spin-orbit term) and the deformed Coulomb potential. The bound and resonant eigenstates of this potential are labeled by the parity quantum number $\pi$ and the projection $\Omega$ of the single particle angular momentum on the symmetry axis. For convenience, deformed Nilsson orbits are denoted by the asymptotic quantum numbers $\left[N n_{z} \Lambda\right]$. Energies and wave functions of the bound (or quasibound) Nilsson orbits can be accurately determined by diagonalizing the deformed Hamiltonian $h$ in an axially deformed harmonic oscillator basis by using the code WSBETA [2].

However the diagonalization results in a large number of spurious positive energy states and does not give information on the widths of the resonances. Therefore, to calculate the complex energy Gamow resonances of the deformed Hamiltonian, we expand the wave function into spherical partial waves $(\{l j\} \equiv i)$

$$
\psi^{\Omega, \pi}(\boldsymbol{r})=\sum_{l=l_{\min }}^{l_{\max }} \sum_{j=\Omega}^{j_{\max }} \frac{u_{l, j}(r)}{r} \mathcal{Y}_{j l \Omega}(\hat{r}) .
$$

The radial wave functions $u_{i}(r)$ can be obtained from a system of coupled differential equations:

$$
u_{i}^{\prime \prime}=\sum_{i^{\prime}}\left[\frac{l_{i}\left(l_{i}+1\right)}{r^{2}} \delta_{i, i^{\prime}}+V_{i, i^{\prime}}(r)-k^{2} \delta_{i, i^{\prime}}\right] u_{i^{\prime}}(r) .
$$

The functions $u_{i}(r)$ are regular at the origin $\left(u_{i}(0)=0\right)$, and they satisfy the purely outgoing boundary condition of Eq. (1) at large distances, $r>$ 
$r_{a s}$, where the coupling terms vanish. The values of $k_{n}$ and $\eta_{n}$ are the same in each channel $i=1, \ldots, n_{c}$. The eigenvalues $k^{2}$ and the normalized eigenfunctions are determined iteratively by integrating the coupled system (3) numerically by using the powerful piecewise perturbation method [3]. Since the solution is diverging at large distances for real values of $r$, to normalize the wave functions, one has to use the regularization procedure. The code we developed for the calculation of the resonant Nilsson state is called CCGAMOW. In its extended precision mode, CCGAMOW is able to calculate the imaginary part of the $\mathcal{E}_{n}$ with an accuracy of $10^{-24} \mathrm{MeV}$.

The transformation to the laboratory system restores the rotational invariance of the Hamiltonian. In the adiabatic approximation, one neglects the rotational motion of the core and the resulting Coriolis coupling. In this approximation, all the members of the ground state rotational band in the daughter nucleus are degenerate (i.e., the moment of inertia $\mathcal{I}=\infty$ ). This is usually referred to as the strong coupling scheme, and the resulting resonant state is called a resonant Nilsson orbit or a Nilsson-Gamow state. Since the spectroscopic factor for a proton emission from a one-quasiparticle state can be expressed as $\mathcal{S}=u_{d}^{2}$ with $u_{d}^{2}$ being calculated in the BCS approximation, the total width becomes $\Gamma=u_{d}^{2} \Gamma_{s p}$.

\section{Deformed Gamow states in a non-adiabatic approach}

It is more appropriate to consider the process of proton emission in the laboratory system of reference. This is a non-adiabatic (NA) approach or a weak coupling approach in which the effect of the Coriolis coupling is implicitely included [4]. Since the moment of inertia of the daughter nucleus $\mathcal{I}$ is finite, the degeneracy of the daughter states is removed: $\epsilon_{I}=\kappa I(I+1)$ $\left(\kappa=\frac{\hbar^{2}}{2 \mathcal{I}}\right)$. The Hamiltonian of the parent nucleus can be expressed as the sum of that representing the rotating core, $H_{0}(\xi)$, and that of a single proton:

$$
H=H_{0}(\xi)+t+\sum_{\lambda} v^{\lambda}(r)\left\{Q_{\lambda}(\xi) \cdot Y_{\lambda}(\hat{r})\right\} .
$$

The wave function of the decaying parent state is characterized by the total angular momentum $J$ and its projection $M$ :

$$
\Psi^{J M}(\vec{r}, \xi)=\frac{1}{r} \sum_{I j l} g_{I j l}^{J}(r) \Phi_{I j l}^{J M}(\hat{r}, \xi),
$$

where

$$
\Phi_{I j l}^{J M}(\hat{r}, \xi)=\sum_{m, \mu}\langle j m I \mu \mid J M\rangle \mathcal{Y}_{j l m}(\hat{r}) \psi_{I, \mu}(\xi)
$$

is the channel function, $\psi_{I, \mu}(\xi)$ is the wave function of the core, and $g_{\alpha}(r)$ are the radial amplitudes. In the following, $\alpha=\{I, j, l\}$ denotes the chan- 
nel index. The radial amplitudes satisfy the system of coupled differential equations

$$
\begin{gathered}
{\left[-\frac{\hbar^{2}}{2 \mu}\left(\frac{d^{2}}{d r^{2}}-\frac{l(l+1)}{r^{2}}\right)+\epsilon_{I}-E\right] g_{\alpha}^{J}(r)} \\
+\sum_{\lambda \alpha^{\prime}} v_{\lambda}(r) \mathcal{V}_{\alpha, \alpha^{\prime}}^{J}(\lambda) g_{\alpha^{\prime}}^{J}(r)=0
\end{gathered}
$$

and are regular in the origin $g_{\alpha}(0)=0$ and should satisfy the outgoing boundary condition at $r=r_{a s}$ :

$$
g_{I j l}\left(r_{a s}\right) \sim O_{l}\left(\eta_{I}, k_{I} r_{a s}\right),
$$

where

$$
\eta_{I}=\frac{Z e^{2} \mu}{\hbar^{2} k_{I}} \quad \text { and } \quad k_{I}^{2}=\frac{2 \mu}{\hbar^{2}}\left(E-\epsilon_{I}\right)=\frac{2 \mu}{\hbar^{2}} Q_{I} .
$$

This boundary condition differs from the one in the adiabatic case because the correct energy of the daughter state $Q_{I}$ is used. The coupled channel non-adiabatic code NONADI is a modified version of CCGAMOW.

In order to obtain widths that can be related to experimental data, it is essential that the resonance should be placed at the proper energy corresponding to the $Q_{p}$ value of the transition. Therefore, in the corrected adiabatic approximation (ADC) $[5,6]$, one readjusts the depth of the Woods-Saxon well $V_{0}$ in order to reproduce the experimental value of $Q_{I}$ when calculating the partial width $\Gamma_{I}$ for the daughter state $I$. In the non-adiabatic (NA) approach, one can adjust both $V_{0}$ and $\mathcal{I}$ to reproduce the mass of the ground state and the energy of the first excited $2^{+}$state. The partial widths for different daughter states $(I=0,2, \ldots)$ can be calculated using the current expression:

$$
\Gamma_{\alpha}^{J}(r)=i \frac{\hbar^{2}}{2 \mu} \frac{g_{\alpha}^{*}(r) g_{\alpha}(r)-g_{\alpha}^{\prime}(r) g_{\alpha}^{*}(r)}{\sum_{\alpha^{\prime}}^{n} N_{\alpha^{\prime}}(r)}
$$

where the $r$-dependent partial norm is defined as $N_{\alpha}(r)=\int_{0}^{r}\left|g_{\alpha^{\prime}}(x)\right|^{2} d x$ and $\Gamma_{I}^{J}(r)=\sum_{l j} \Gamma_{I l j}^{J}(r)$. The partial widths depend on $r$ and saturate only in the region of large $r$-values where the multipole couplings become negligible. However, the total particle decay width is $r$-independent due to the flux conservation: $\Gamma^{J}=\sum_{\alpha}^{n} \Gamma_{\alpha}^{J}(r)=\sum_{I} \Gamma_{I}^{J}(r)$. The saturated values $\left|c_{\alpha}\right|^{2}=N_{\alpha}\left(r_{a s}\right)$ characterize the wave function of the non-adiabatic Gamow resonance. From the partial widths, one can determine the branching ratios $B(I)=\frac{\Gamma_{I}^{J}(r)}{\Gamma^{J}}$ for the decay to excited states in the daughter nucleus of the daughter states (fine structure in proton decay). These quantities can be calculated not only for the ground state (or isomeric state) of the parent nucleus but also for the members of the corresponding rotational bands. 
The energies and wave functions of these band members are affected by the Coriolis coupling.

\section{Results}

The first nonadiabatic (NA) calculations for deformed proton emitters was carried out by Kruppa et al. [7] in their analysis of the fine structure in ${ }^{131} \mathrm{Eu},{ }^{141} \mathrm{Ho}$, and ${ }^{141 m} \mathrm{Ho}$. A detailed comparison of the NA and the ADC approaches for several deformed proton emitters can be found in Ref. [8]. A general comparison of NA and ADC results for the deformed proton emitters ${ }^{109} \mathrm{I},{ }^{113} \mathrm{Ce},{ }^{117} \mathrm{La},{ }^{131} \mathrm{Eu},{ }^{141} \mathrm{Ho}$, and ${ }^{141 m} \mathrm{Ho}$ is given in Table 4. Though in some cases NA and ADC results are very close (e.g., for the

TABLE 1. Comparison of NA and ADC approaches for half-lives and branching ratios $B(2)$ for several deformed proton emitters

\begin{tabular}{ccccc}
\hline Parent & Orbit & $T_{\frac{1}{2}}^{A D C} / T_{\frac{1}{2}}^{N A}$ & $B(2)^{N A}$ & $B(2)^{A D C}$ \\
\hline${ }^{109} \mathrm{I}$ & {$[420] \frac{1}{2}$} & 0.25 & $0 \%$ & $0 \%$ \\
${ }^{113} \mathrm{Cs}$ & $J=\frac{3}{2}{ }^{+}$ & 0.20 & $0 \%$ & $0 \%$ \\
${ }^{117} \mathrm{La}$ & {$[422] \frac{3}{2}$} & 0.88 & $3 \%$ & $0.3 \%$ \\
${ }^{131} \mathrm{Eu}$ & {$[411] \frac{3}{2}$} & 1.43 & $39 \%$ & $37 \%$ \\
${ }^{141} \mathrm{Ho}$ & {$[523] \frac{7}{2}$} & 0.35 & $6 \%$ & $2.7 \%$ \\
${ }^{141 m} \mathrm{Ho}$ & {$[411] \frac{1}{2}$} & 1.04 & $1 \%$ & $1 \%$ \\
\hline
\end{tabular}

${ }^{141 m}$ Ho [411] $\frac{1}{2}$ resonance), there are large differences for most of the nuclei studied. In the case of the ${ }^{113} \mathrm{Cs} J=\frac{3}{2}^{+}$state, the Nilsson labeling loses its validity due to the strong $K$-mixing.

The largest difference between NA and ADC is obtained in the case of ${ }^{141}$ Ho. The parent state, labeled as $[523] \frac{7^{-}}{}{ }^{-}$, originates from the $h_{\frac{11}{2}}$ spherical shell. The $h_{\frac{11}{2}}$ component is still the dominant one at the calculated ground state deformation $\beta_{2}=0.29, \beta_{4}=-0.06$. The $7 / 2^{-} \rightarrow 0^{+}$transition can only proceed via the $f_{7 / 2}$ partial wave, which is very sensitive to the small $\left|c_{0,3, \frac{7}{2}}\right|^{2}$ component of the wave function. The use of the NA approach reduces this component to 0.011 from 0.027 in the AD case, which results in the reduction of $\Gamma_{0}+$ and an increase of $B(2)$ to $6 \%$.

The decay from the ${ }^{141 m}$ Ho isomeric state, which is identified as a [411] $\frac{1}{2}$ Nilsson-Gamow state, is interesting since it is associated with the strong Coriolis perturbation. Namely, in the NA approach, the $J=1 / 2$ and $J=3 / 2$ levels are almost degenerate, and the $J=5 / 2$ and $J=7 / 2$ levels also form a doublet. 
In ${ }^{131} \mathrm{E} 1$, the highest branching ratio, $B(2)=0.52$, was calculated for the yet-unobserved transition from the $[532] \frac{5}{2}^{-}$resonant orbit. In the NA approach, a nonzero value of $\left|c_{2 p_{3 / 2}}\right|^{2}=0.003$ is due to the Coriolis coupling, while it is obviously zero in the ADC approach when $K=\Omega=5 / 2$. For the $\frac{5}{2}^{-} \rightarrow 2^{+}$transition, the lowest partial wave in the NA case is $p_{3 / 2}$, but it is $l \geq 3$ in ADC. The lower the $l_{\text {min }}$ value, the larger the penetrability, and this magnifies the role of the $p_{3 / 2}$ component (which is responsible for $15 \%$ of the total width $\Gamma$ ).

\section{Conclusions}

We demonstrated that it is possible to calculate the complex energy of the deformed Gamow state with a precision that is high enough so that the halflife calculated from the imaginary part of the energy is meaningful. We also performed a comparison between non-adiabatic and adiabatic calculations. It can be concluded that, in many cases, the corrected adiabatic treatment cannot be used as a substitute for the full non-adiabatic calculations.

Acknowledgement. This work was supported in part by the Hungarian NRF (OTKA T026244 and T029003) and by the U.S. Department of Energy under Contract Nos. DE-FG02-96ER40963 (University of Tennessee), DE-FG05-87ER40361 (Joint Institute for Heavy Ion Research), and DEAC05-00OR22725 with UT-Battelle, LLC (Oak Ridge National Laboratory).

\section{References}

1. Åberg, S., Semmes, P.B., and Nazarewicz, W. (1997) Phys. Rev. C56, pp. 1762-1773

2. Cwiok, S., Dudek, J., Nazarewicz, W., Skalski, J., and Werner, T. (1987) Comput. Phys. Commun. 46, pp. 379-399

3. Ixaru, L. Gr. (1984) Numerical Methods for Differential Equations, (Reidel, Dordrecht)

4. Tamura, T. (1965) Rev. Mod. Phys. 67, p. 679

5. Sonzogni, A.A. et al. (1999) Phys. Rev. Lett. 83, p. 1116

6. Maglione, E., Ferreira, L.S., and Liotta, R.J. (1998) Phys. Rev. Lett. 81, p. 538; (1999) Phys. Rev. C59, R589

7. Kruppa, A. T., Barmore, B., Nazarewicz, W., and Vertse, T. (2000) Phys. Rev. Letts. 84, pp. 4549-4552

8. Barmore, B., Kruppa, A. T., Nazarewicz, W., and Vertse, T. (2000) Phys. Rev. C62, 054315 pp 1-12 